\title{
Perceptions of Resident Physicians About Management of Inpatient Hyperglycemia in an Urban Hospital
}

\author{
Vasundhara Cheekati, MD ${ }^{1}$ \\ Robert C. Osburne, MD $^{1}$ \\ Kimberly A. Jameson ${ }^{2}$ \\ Curtiss B. Cook, $\mathrm{MD}^{3}$ \\ ${ }^{1}$ Division of Endocrinology, Atlanta Medical Cen- \\ ter, Atlanta, Georgia. \\ ${ }^{2}$ Section of Practice Analysis, Mayo Clinic, \\ Scottsdale, Arizona. \\ ${ }^{3}$ Division of Endocrinology, Mayo Clinic, Scotts- \\ dale, Arizona.
}

BACKGROUND: Information regarding practitioner beliefs about inpatient diabetes care is limited.

OBJECTIVE: To assess resident physician attitudes about inpatient hyperglycemia and determine perceived barriers to optimal glycemic control in an urban hospital setting.

DESIGN: A previously developed questionnaire was modified and administered. Residents were asked about the importance of inpatient glucose control, desirable glucose ranges, and problems encountered when managing hyperglycemia.

SETTING: Urban teaching hospital.

RESULTS: Of 85 resident physicians, 66 completed the survey (mean age, 31 years; $47 \%$ men; $33 \%$ in first residency year). Most respondents categorized glucose control as "very important" in critically-ill and perioperative patients but only "somewhat important" in non-critically-ill patients. Most residents said they would target a therapeutic glucose range within the recommended levels. Most residents (88\%) also said they felt "very comfortable" or "somewhat comfortable" using subcutaneous insulin therapy, whereas some were "not at all comfortable" with either subcutaneous (11\%) or intravenous (18\%) administration. In general, respondents were not very familiar with existing institutional policies and preprinted order sets. The most commonly reported barrier to management of inpatient hyperglycemia was lack of knowledge about appropriate insulin regimens and their use. Anxiety about hypoglycemia was only the third most frequent concern.

CONCLUSION: Most residents acknowledged the importance of good glucose control in hospitalized patients and chose target glucose ranges consistent with existing guidelines. Lack of knowledge about insulin treatment options was the most commonly cited barrier to ideal management. Educational programs should emphasize inpatient treatment strategies for glycemic control. Journal of Hospital Medicine 2009;4:E1-E8. ㅇ 2009 Society of Hospital Medicine.

KEYWORDS: diabetes, hospitalizations, medical education, practitioner attitudes.

0 ngoing surveillance indicates that the number of hospitalizations involving patients with a diagnosis of diabetes mellitus is increasing in the United States. ${ }^{1,2}$ Hospitalized patients with hyperglycemia have worse outcomes (eg, greater mortality, longer length of stay, and more infections) than those without high glucose levels. ${ }^{3,4}$ The rate of adverse outcomes associated with hyperglycemia can be decreased with improved management. ${ }^{3,4}$ Consequently, the American Diabetes Association and the American College of Endocrinology advocate lower glucose targets for 
all hospitalized patients regardless of whether they have a known diagnosis of diabetes. ${ }^{3,4}$

Practitioners continue to debate the exact glucose targets that should be attained for inpatients; ${ }^{5,6}$ however, there is more to inpatient hyperglycemia management than just trying to achieve a specific glucose range. Caring for patients with diabetes in the hospital is complex and must also encompass patient safety, but many practitioners perceive a state of "glycemic chaos" in the hospital. ${ }^{7}$ Because many physicians frequently overlook diabetes and glucose control in the hospital, appropriate therapeutic responses to hyperglycemia do not occur. ${ }^{8-10}$ National, ${ }^{11,12}$ state $^{13}$ and specialty societies ${ }^{3,4,14}$ are working to improve care for hospitalized patients with hyperglycemia. A recent consensus conference emphasized the need to develop broad-based educational programs to increase awareness about the importance of inpatient glycemic control and to develop a standardized set of tools for hospitals to use to improve care. ${ }^{4}$ However, there is ongoing concern about the slow pace at which hospitals are implementing recommendations about glycemic control. ${ }^{4}$

Intensive and prolonged educational efforts about the importance of glycemic control will be essential ingredients of any quality improvement effort designed to create "glycemic order" out of "glycemic chaos" in the hospital. ${ }^{15}$ Before educational interventions and policies directed at improving the management of hyperglycemia in hospitalized patients can be developed, institutions need to gain a better understanding of how clinicians view the importance of inpatient glucose control and which barriers they perceive as constraints to their ability to care for inpatients with hyperglycemia.

At Atlanta Medical Center (AMC), the large urban teaching hospital where this study was conducted, the glucose control team detected resistance to changes that were implemented to improve the hospital's quality of glycemic control ${ }^{16}$ this observation led to a desire to gain more information about practitioner attitudes regarding inpatient glucose control management. Data on practitioner attitudes and beliefs about inpatient hyperglycemia are only now emerging and are limited to studies from a single institution. ${ }^{17,18}$ Thus, additional studies are needed to determine whether findings from these first studies are applicable to other types of hospital set- tings that have different inpatient populations. To gain additional insight into clinician beliefs about inpatient glucose control, we adapted a previously published questionnaire ${ }^{17,18}$ and used it to survey resident physicians training at AMC.

\section{METHODS \\ Setting}

AMC is a community teaching hospital located in downtown Atlanta, Georgia, and it is a 460-bed tertiary care facility. All adult general medical and surgical specialties are represented, in addition to obstetrics and gynecology, a neonatal unit, a level 2 trauma unit, a stroke unit, and an inpatient rehabilitation unit. The inpatient population is mostly minority in mix, with $45 \%$ African American, $37 \%$ Caucasian, $12 \%$ Hispanic, and $6 \%$ other races. ${ }^{16,19}$ Various types of practitioners provide patient care at AMC, including postgraduate trainees (resident physicians), graduate medical education faculty, physician assistants, and nurse practitioners.

\section{Description of Survey}

A previously published survey instrument (the Mayo Clinic Inpatient Diabetes Attitude Survey) was adapted for this project. ${ }^{17,18}$ The survey was developed by a team of endocrinologists and primary care physicians with the assistance of our institutional experts in survey design; it was then piloted and submitted to iterative cycles of review and revision. ${ }^{17}$ The survey was used to assess beliefs first among resident physicians ${ }^{17}$ and then among midlevel practitioners at the same facility. ${ }^{18}$ The survey tool was specifically developed to evaluate perceptions of practitioners about inpatient glucose management, including beliefs about the importance of glucose control in the hospital, optimal glucose targets, and barriers to the successful treatment of hyperglycemia. Additionally, the questionnaire was previously used to solicit residents' perceptions about the proportion of their inpatient practices represented by diabetes patients, their beliefs regarding whether patients were achieving their glucose targets, their degree of comfort with managing hyperglycemia and using insulin therapy, and their familiarity with existing institutional policies and preprinted insulin order sets. ${ }^{17,18}$ The questionnaire was expanded from its original version to include questions about the use of intravenous insulin. 


\section{Survey Participants}

As in most academic teaching facilities, at AMC resident physicians treat many of the inpatients who have a diagnosis of diabetes, making the residents an important group to target for educational programs focused on inpatient glucose management. Thus, the audience for this survey included only resident physicians who had ongoing inpatient responsibilities so that the data could be used to assist in educational planning strategies for increasing awareness and improving treatment of inpatients with hyperglycemia. We identified 85 residents who met the inclusion criteria, and we conducted the survey from March to April 2007.

\section{Data Analysis}

Written surveys were collected and entered into SurveyTracker version 4.0 (Training Technologies, Inc., Lebanon, Ohio) for analysis. We then examined the distribution of responses to individual questions. Finally, we listed the frequency of expressed barriers to inpatient glucose management from most common to least common.

\section{RESULTS \\ Respondent Demographics}

Sixty-six of 85 residents (78\%) responded to the survey. The mean age of the respondents was 31 years, $47 \%$ were men, $33 \%$ were in their first year of residency training, and $73 \%$ had graduated from medical school during the year 2002 or later. The residents represented the following departments: $41 \%$ from internal medicine $(n=27), 18 \%$ from family medicine $(\mathrm{n}=12), 15 \%$ from general surgery $(\mathrm{n}=10), 12 \%$ from orthopedics $(\mathrm{n}=8)$, and $14 \%$ from obstetrics and gynecology $(\mathrm{n}=9)$.

\section{Perceptions About Inpatient Burden of Diabetes}

When asked to estimate the percentage of their hospitalized patients who were considered to have a diagnosis of diabetes or hyperglycemia, $14 \%$ of the residents indicated that $0 \%$ to $20 \%$ of their hospitalized patients were in this category, $30 \%$ of the residents estimated that $21 \%$ to $40 \%$ of their inpatients were in this group, and $25 \%$ of the residents believed that such a diagnosis applied to $41 \%$ to $60 \%$ of their inpatients. Additionally, $23 \%$ of the residents said that $61 \%$ to $80 \%$ of their inpatients were considered to have a diagnosis of diabetes or hyperglycemia, $3 \%$ of the residents estimated that $81 \%$ to $100 \%$ of their hospitalized patients had such a diagnosis, and $5 \%$ of the residents were unsure. Thus, respondents perceived that diabetes constituted a substantial portion of their inpatient practices, with $50 \%$ of the residents estimating that more than $40 \%$ of their hospitalized patients had a diagnosis of diabetes or hyperglycemia and nearly $25 \%$ of responding residents believing that more than $60 \%$ of their inpatients had the same diagnosis.

\section{Views on the Importance of Glycemic Control}

Most resident physicians believed that good glycemic control was important in hospitalized patients (Table 1); 97\% believed that it was "very important" to have tight glycemic control in critically-ill patients, $72 \%$ emphasized that it was "very important" in non-critically-ill patients, and $85 \%$ indicated that it was "very important" during the perioperative period. Nearly all residents believed that it was important to achieve good glycemic control in pregnant patients (Table 1).

\section{Comfort With Treatment and Management}

Survey participants were asked how comfortable they felt about different scenarios pertaining to inpatient glucose management (Table 1). Although more than $40 \%$ of respondents indicated that they felt "very comfortable" treating hyperglycemia and hypoglycemia in the hospital, a large proportion (50\% or more) also indicated that they were only "somewhat comfortable" or "not at all comfortable" treating these conditions. Similarly, in response to questions about their degree of comfort working with subcutaneous or intravenous insulin, more than $50 \%$ of trainees were only "somewhat comfortable" or "not at all comfortable." Finally, most were "not at all comfortable" with the use of insulin pumps in the hospital (Table 1).

\section{Familiarity With Existing Policies and Procedures}

Most of the trainees indicated that they were "not at all familiar" with existing hospital policies and orders pertaining to insulin pumps (Table 1). Most respondents were only "somewhat familiar" with the institutional hypoglycemia policy, but a substantial percentage $(32 \%)$ were either "not at all familiar" or even unaware that an institutional hypoglycemia policy existed. Similarly, most were only "somewhat familiar," "not at all familiar," or even unaware of orders or policies pertaining to 
TABLE 1

Summary of Resident Physicians' Opinions About Inpatient Hyperglycemia

\begin{tabular}{|c|c|c|c|c|}
\hline \multirow{2}{*}{$\frac{\text { Category }}{\text { Importance of Treating }}$} & \multicolumn{4}{|c|}{ Response } \\
\hline & Very Important & Somewhat Important & Not at All Important & Don't Know \\
\hline Critically ill patients & 97 & 3 & 0 & 0 \\
\hline Non-critically-ill patients & 72 & 26 & 2 & 0 \\
\hline Perioperative patients & 85 & 15 & 0 & 0 \\
\hline Pregnant patients & 97 & 3 & 0 & 0 \\
\hline Comfort Level & Very Comfortable & Somewhat Comfortable & Not at All Comfortable & Don't Know \\
\hline Treating hyperglycemia & 42 & 47 & 11 & 0 \\
\hline Treating hypoglycemia & 49 & 44 & 6 & 1 \\
\hline Using subcutaneous insulin & 44 & 44 & 11 & 1 \\
\hline Using insulin drips & 38 & 42 & 18 & 2 \\
\hline Using insulin pumps* & 14 & 17 & 58 & 12 \\
\hline Familiarity & Very Familiar & Somewhat Familiar & Not at All Familiar & Unaware of Policy \\
\hline Insulin pump policy & 9 & 15 & 52 & 24 \\
\hline Insulin pump orders & 6 & 17 & 54 & 23 \\
\hline Hypoglycemia policy & 23 & 45 & 17 & 15 \\
\hline Subcutaneous insulin orders & 34 & 35 & 14 & 17 \\
\hline Intravenous insulin orders & 38 & 33 & 21 & 8 \\
\hline Glucose Goal, mg/dL & $80-110$ & $111-180$ & $181-250$ & Don't Know \\
\hline Critically ill patients & 91 & 6 & 2 & 1 \\
\hline Non-critically-ill patients & 53 & 46 & 0 & 1 \\
\hline Perioperative patients & 76 & 23 & 0 & 1 \\
\hline $\begin{array}{l}\text { Glucose Level for Initiation } \\
\text { of IV Insulin, mg/dL }\end{array}$ & $>110$ & $>140$ & $>180$ & Don't Know \\
\hline Critically ill patients & 8 & 30 & 51 & 11 \\
\hline Non-critically-ill patients & 1 & 6 & 65 & 28 \\
\hline Pregnant patients & 8 & 27 & 30 & 35 \\
\hline $\begin{array}{l}\text { NOTE: Data are percentage of total } \\
\text { Abbreviation: IV, intravenous. } \\
{ }^{*} \text { Total percentage exceeds } 100 \% \text { due }\end{array}$ & & & & \\
\hline
\end{tabular}

use of subcutaneous or intravenous insulin (Table 1).

\section{Beliefs About Glucose Targets and Hypoglycemia}

When asked to indicate the target glucose levels that they would like to achieve, most resident physicians indicated that good glycemic control meant a target range of 80 to $110 \mathrm{mg} / \mathrm{dL}$ for critically-ill patients and for perioperative patients. For non-critically-ill patients, targets were split between a target range of 80 to $110 \mathrm{mg} / \mathrm{dL}$ and 111 to $180 \mathrm{mg} / \mathrm{dL}$. Trainees rarely suggested targets greater than $180 \mathrm{mg} / \mathrm{dL}$ (Table 1).

Most respondents believed that they were achieving their glycemic goals in $41 \%$ to $60 \%$ of their patients (Fig. 1A). More than half (56\%) perceived that they were achieving their glucose targets in more than $40 \%$ of their diabetes 

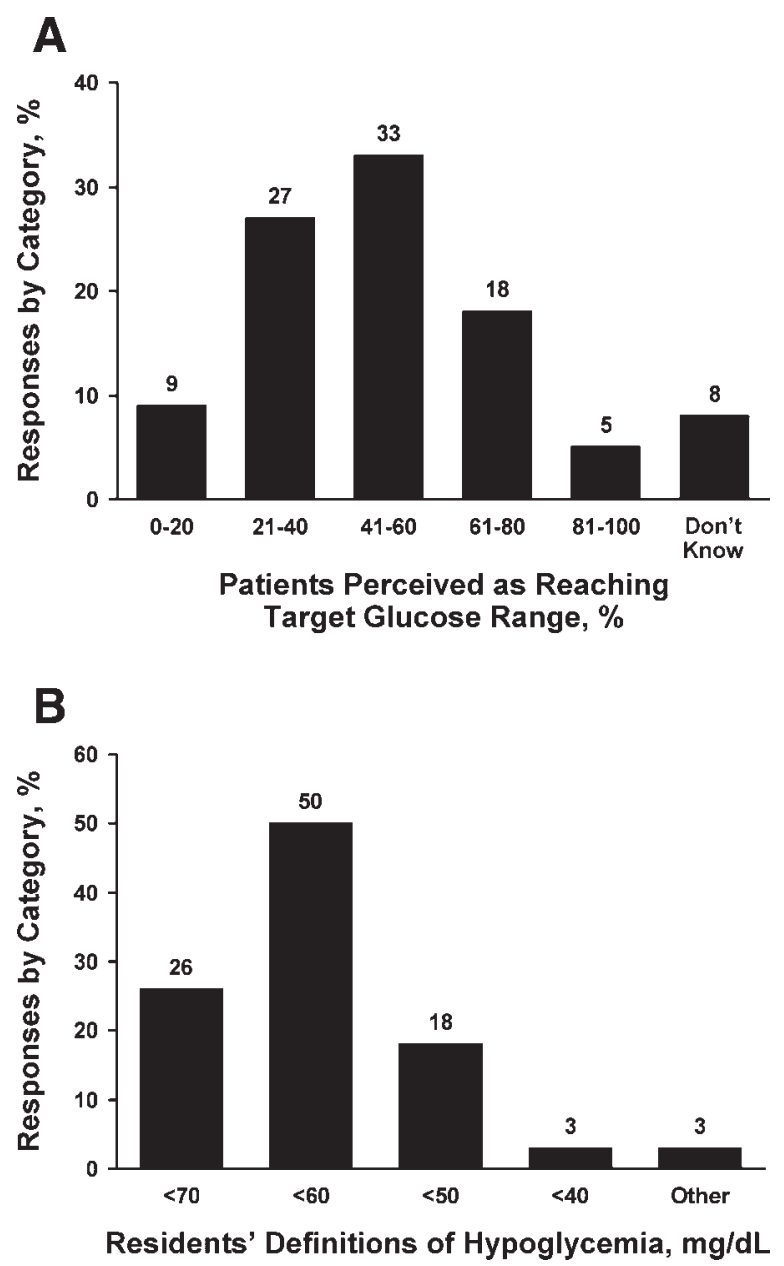

FIGURE 1. Summary of questionnaire responses, showing resident physicians' perceptions about (A) how many of their inpatients were achieving desired glucose goals and (B) the glucose levels the residents used to define hypoglycemia. (A) Most respondents believed that they were achieving their glycemic goals in $41 \%$ to $60 \%$ of their patients. More than half $(56 \%)$ perceived that they were achieving their glucose targets in more than $40 \%$ of their diabetes patients. (B) When asked at what glucose level they first considered the patient to be hypoglycemic, half of the respondents chose a value of $<60 \mathrm{mg} / \mathrm{dL}$, and $21 \%$ used an even lower cutoff of $<50 \mathrm{mg} / \mathrm{dL}$ for a diagnosis of hypoglycemia.

patients. When asked at what glucose level they first considered the patient to be hypoglycemic, half of the respondents chose $<60 \mathrm{mg} / \mathrm{dL}$ (Fig. $1 B)$, although some had even lower cutoffs before they considered someone to have a diagnosis of hypoglycemia.

\section{Thresholds for Starting Intravenous Insulin}

For both critically-ill and non-critically-ill patients, most resident physicians indicated that they would wait until the glucose level was greater than $180 \mathrm{mg} / \mathrm{dL}$ before starting an insulin infusion (Table 1). Likewise, obstetrics residents identified a glucose level greater than $180 \mathrm{mg} / \mathrm{dL}$ as a threshold to start intravenous insulin in pregnant patients.

\section{Perceived Barriers to Care}

The survey concluded with a question that asked resident physicians to choose from a list of factors they perceived as obstacles to inpatient glucose management. The 5 most frequently chosen obstacles, from most common to least common, were as follows: "knowing what insulin type or regimen works best," "fluctuating insulin demands related to stress" and "risk of causing patient hypoglycemia" (cited with equal frequency), "unpredictable changes in patient diet and meal times," and "unpredictable timing of patient procedures" (Table 2).

\section{DISCUSSION}

In recent years national and regional organizations have focused greater attention on the management of hyperglycemia among inpatient populations by introducing and promoting guidelines for better care., ${ }^{3,4,11-14}$ A consensus conference in 2006 urged hospitals to "move rapidly to make euglycemia a goal for all inpatients and to make patient safety in glycemic control a reality... . ${ }^{20}$ AMC has already taken some steps toward understanding and improving its hospitalbased care of hyperglycemia, including understanding the mortality associated with hyperglycemia within the institution and implementing a novel insulin infusion algorithm. ${ }^{16,19}$

Before hospitals can develop high-quality improvement and educational programs focused on inpatient hyperglycemia, they will need more insight into their clinicians' views on inpatient glycemic control and the perceived barriers to successful treatment of hyperglycemia. However, the only data that have been published about practitioner attitudes on inpatient diabetes and glycemic control are from a single institution. ${ }^{17,18}$ Thus, analyses should be broadened to include different types of hospital settings to determine common beliefs on the topic.

AMC is very different from the hospital facility where earlier studies on physician attitudes about inpatient glucose management were conducted. 
TABLE 2

Resident Physicians' Perceived Barriers to Management of Inpatient Hyperglycemia

\begin{tabular}{ll}
\hline & $\begin{array}{l}\text { Response, } \\
\text { Number (\%) } \\
\text { (n = 66) }\end{array}$ \\
Barrier & $26(39)$ \\
\hline Knowing what insulin type or regimen works best & $26(39)$ \\
Fluctuating insulin demands related to stress/concomitantly & \\
$\quad$ used medications & $25(38)$ \\
Risk of causing hypoglycemia & $25(38)$ \\
Unpredictable changes in patient diet and mealtimes & $19(29)$ \\
Unpredictable timing of patient procedures & $18(27)$ \\
Patient not in hospital long enough to control glucose & $18(27)$ \\
$\quad$ adequately & $16(24)$ \\
Shift changes and cross-coverage lead to inconsistent & $14(21)$ \\
$\quad$ management & $14(21)$ \\
Knowing best options to treat hyperglycemia & $13(20)$ \\
Knowing when to start insulin & $11(17)$ \\
Knowing how to adjust insulin & $10(15)$ \\
Conversion between different forms of insulin & $10(15)$ \\
Lack of guidelines on how to treat hyperglycemia & $7(11)$ \\
Preferring to defer management to outpatient care or to another & $7(11)$ \\
specialty & $6(9)$ \\
Knowing how to start insulin & $6(9)$ \\
Knowing how to best prevent hypoglycemia & $4(6)$ \\
Treating hyperglycemia is not a priority in the hospital & $3(5)$ \\
Other* & \\
Disagreement with other team members on how to control & \\
\hline & \\
\hline & \\
\hline
\end{tabular}

NOTE: Itemized from most to least frequently cited.

${ }^{*}$ Nonavailability of intravenous insulin out of the intensive care unit; nurses not following orders for insulin.

Whereas the site of the earlier studies is located in the Southwest and has a diabetes inpatient population that is primarily white, AMC is an urban hospital in the Southeast whose diabetes inpatient population is primarily minority. ${ }^{21}$ Despite the institutional, geographic, and patient population differences, however, results of the current survey suggest that there may be similar beliefs among practitioners about inpatient glucose management as well as common knowledge deficits that can be targeted for educational interventions.

Similar to the resident physicians surveyed in previous studies, ${ }^{17,18}$ AMC resident physicians considered diabetes to be a substantial part of their inpatient practices: $56 \%$ of respondents believed that more than $40 \%$ of their inpatients had a diagnosis of diabetes. Historically, the prevalence at AMC of hyperglycemia has been about $38 \%$ and the prevalence of diabetes about $26 \% .{ }^{19}$
The increasing number of hospital dismissals attributable to diabetes likely has increased the inpatient prevalence of the disease at AMC as well, but very high rates perceived by some residents (eg, $81 \%-100 \%$ ) are likely not accurate. Nonetheless, this perception of such a large burden of diabetes clearly substantiates the need to provide pertinent information and essential tools to clinicians for successful management of hyperglycemia in hospital patients. We also established that most AMC resident physicians who were surveyed believed that good glucose control was "very important" in situations relating to critical illness or noncritical illness. For most respondents, good glucose control was also "very important" in the perioperative period. This finding suggests that the trainees understand the importance of good glucose control in such situations.

In keeping with findings from previous studies, ${ }^{17,18}$ respondents to this survey indicated glucose targets that would be well within currently existing guidelines. ${ }^{3,4}$ Glucose management training might be improved by conveying whether actual glucose outcomes match residents' perceived achievement of glycemic control.

Insulin is the recommended treatment for inpatient hyperglycemia, ${ }^{3,4}$ yet residents' responses reflected concern about insulin use. The most commonly noted issues, cited with equal frequency, were related to insulin use: "knowing what insulin type or regimen works best" and "fluctuating insulin demands related to stress/concomitantly used medications." Our survey did not evaluate whether residents had different degrees of comfort with different subcutaneous insulin programs (eg, sliding scale versus basal-bolus). Future surveys could be modified to better hone in on evaluating self-perceived competencies in these areas.

Given the increasing complexity of insulin therapy, resident physicians' perception of insulin administration as the top barrier to inpatient glucose management may not be surprising. ${ }^{17,18}$ The number of insulin analogs has increased in recent years. Moreover, numerous intravenous insulin algorithms are available. ${ }^{22,23}$ Errors in insulin administration are among the most frequently occurring medication errors in hospitals. ${ }^{24}$ To address patient safety and medical system errors in the fields of diabetes and endocrinology, the American College of Endocrinology published a position statement on the topic in $2005 .{ }^{25}$ Guidelines about when to initiate insulin therapy, how 
to choose from numerous insulin treatment options, and how to adjust therapy in response to rapidly changing clinical situations will have to be integrated into any effort to improve inpatient glucose management. One study indicated that an educational process focused on teaching residents about insulin therapy can be successful. ${ }^{26}$

Clinician fear of hypoglycemia is often perceived as the primary obstacle to successful control of inpatient glucose levels; ${ }^{3,27}$ however, this was not the chief concern expressed by either AMC resident physicians or by practitioners surveyed in prior studies. ${ }^{17,18}$ Emerging data suggest that hypoglycemia in the hospital is actually uncommon. ${ }^{21,28}$ As hospitals intensify hyperglycemia management efforts, hypoglycemia and concerns about its frequency of occurrence will most likely increase. No consensus exists regarding the number of hypoglycemic events that are acceptable in a hospitalized patient. The American Diabetes Association Workgroup on Hypoglycemia has defined hypoglycemia as an "(arterialized venous) plasma glucose concentration" of less than or equal to $70 \mathrm{mg} / \mathrm{dL}^{29}$ As a group, residents surveyed for the current study were not consistent in their definition of hypoglycemia.

The residents at AMC also reported potential obstacles to care besides insulin management that suggest system-based problems. "Unpredictable timing of patient procedures" and "unpredictable changes in patient diet and mealtimes" were among the 5 most frequently cited concerns. Other concerns included "patient not in hospital long enough to adequately control glucose" and "shift changes and cross-coverage lead to inconsistent management." These findings are identical to those of prior studies ${ }^{17,18}$ and suggest systembased problems as common barriers to inpatient glucose management. Some of these obstacles, such as length of hospital stay and timing of procedures, would be difficult to reengineer. However, other aspects, such as adjusting therapy to mealtimes and ensuring standardization of treatment across shifts, could be addressed through institution-wide education and changes in policies.

As in previous studies, another major finding that emerged from this survey was the lack of resident physician familiarity with existing policies and procedures related to inpatient glucose management. AMC has a longstanding policy on hypoglycemia management and has preprinted order sets for subcutaneous insulin. AMC has imple- mented a revised insulin infusion algorithm ${ }^{16}$ in addition to a policy and an order set for the use of insulin pumps. ${ }^{30}$ There are no specific data on how many patients receiving insulin pump therapy are hospitalized, but these patients are likely to be encountered only rarely in the hospital setting. Hence, it may not be surprising that residents are unfamiliar with policies pertaining to inpatient insulin pump use, but they should at least be aware that guidance is available. One of the first steps to enhancing and standardizing hospital glucose management may simply be to make certain that clinicians are familiar with policies that are already in place within the institution.

A limitation of this study is the small sample size. The results of the present study should not be extrapolated to nonresident medical staff such as attending physicians, but the questionnaire could be adapted, with minor modifications, to investigate how other health care professionals view inpatient glucose management. In addition, the questionnaire could be used to assess changes in beliefs over time. Future studies should be designed to correlate resident perceptions about their inpatient diabetes care and actual practice patterns.

More surveys such as the one reported on here need to be conducted in additional institutions in order to expand our understanding of practitioner attitudes regarding inpatient diabetes care. Data from the current study and previous ones suggest that practitioners share beliefs, knowledge deficits, and perceived barriers about inpatient glucose management. Most AMC resident physicians recognized the importance of good glucose control and set target glucose ranges consistent with existing guidelines. Knowledge deficits may be addressed by developing training programs that specifically spotlight insulin use in the hospital. As a first step to quality improvement, training programs should focus on familiarizing staff with existing institutional policies and procedures pertaining to hospital hyperglycemia. In addition, hospitals need to design strategies to overcome perceived and actual barriers to care so that they can realize the desired improvement in the management of hyperglycemia in their patients. We have already begun the development and implementation of educational modules directed at addressing many of these important issues. 
Address for correspondence and reprint requests: Robert C. Osburne, MD, Atlanta Diabetes Associates, 77 Collier Road, Suite 2080, Atlanta, GA 30309; Telephone: (404) 355-4393; Fax: (404) 609-7648; E-mail: rosburne@ atlantadiabetes.com

Received 22 January 2008; revision received 26 March 2008; accepted 8 April 2008.

\section{REFERENCES}

1. Centers for Disease Control and Prevention. Hospitalization for diabetes as first-listed diagnosis. Available at: http://www.cdc.gov/diabetes/statistics/dmfirst/index.htm. Accessed October 2008.

2. Centers for Disease Control and Prevention. Hospitalizations for diabetes as any-listed diagnosis. Available at: http://www.cdc.gov/diabetes/statistics/dmany/index.htm. Accessed October 2008.

3. Clement S, Braithwaite SS, Magee MF, et al. Management of diabetes and hyperglycemia in hospitals. Diabetes Care. 2004;27:553-591.

4. ACE/ADA Task Force on Inpatient Diabetes. American College of Endocrinology and American Diabetes Association consensus statement on inpatient diabetes and glycemic control. Endocr Pract. 2006;12:458-468.

5. Inzucchi SE, Rosenstock J. Counterpoint: inpatient glucose management: a premature call to arms? Diabetes Care. 2005;28:976-979.

6. Bryer-Ash M, Garber AJ. Point: inpatient glucose management: the emperor finally has clothes. Diabetes Care. 2005;28:973-975.

7. Umpierrez G, Maynard G. Glycemic chaos (not glycemic control) still the rule for inpatient care: how do we stop the insanity? [Editorial] J Hosp Med. 2006;1:141-144.

8. Levetan CS, Passaro M, Jablonski K, Kass M, Ratner RE. Unrecognized diabetes among hospitalized patients. Diabetes Care. 1998;21:246-249.

9. Knecht LA, Gauthier SM, Castro JC, et al. Diabetes care in the hospital: is there clinical inertia? J Hosp Med. 2006;1: 151-160.

10. Schnipper JL, Barsky EE, Shaykevich S, Fitzmaurice G, Pendergrass ML. Inpatient management of diabetes and hyperglycemia among general medicine patients at a large teaching hospital. J Hosp Med. 2006;1:145-150.

11. The Joint Commission. Inpatient diabetes. Available at: http://www.jointcommission.org/CertificationPrograms/ Inpatient+Diabetes. Accessed October 2008.

12. Institute for Healthcare Improvement. Implement effective glucose control. Available at: http://www.ihi.org/IHI/ Topics/CriticalCare/IntensiveCare/Changes/ImplementEffectiveGlucoseControl.htm. Accessed October 2008.

13. Cook CB, Stockton L, Baird M, et al.; the Georgia Hospital Association Diabetes Special Interest Group. Working to improve care of hospital hyperglycemia through statewide collaboration. Endocr Pract. 2007;13:45-50.

14. Society of Hospital Medicine. Glycemic control resource room. Available at: http://www.hospitalmedicine.org/AM/ Template.cfm?Section=Search_Advanced_Search\&template $=/ \mathrm{CM} / \mathrm{HTMLDisplay} . \mathrm{cfm} \&$ ContentID $=14414$. Accessed October 2008.
15. Cook CB. Author reply to Flansbaum B. Management of hyperglycemia [Correspondence]. J Hosp Med. 2006;1: 383-385.

16. Osburne RC, Cook CB, Stockton L, et al. Improving hyperglycemia management in the intensive care unit: preliminary report of a nurse-driven quality improvement project using a redesigned insulin infusion algorithm. Diabetes Educ. 2006;32:394-403.

17. Cook CB, McNaughton DA, Braddy CM, et al. Management of inpatient hyperglycemia: assessing perceptions and barriers to care among resident physicians. Endocr Pract. 2007;13:117-124.

18. Cook CB, Jameson KA, Hartsell ZC, et al. Beliefs about hospital diabetes and perceived barriers to glucose management among inpatient midlevel practitioners. Diabetes Educ. 2008;34:75-83.

19. Umpierrez GE, Isaacs SD, Bazargan N, You X, Thaler LM, Kitabchi AE. Hyperglycemia: an independent marker of inhospital mortality in patients with undiagnosed diabetes. J Clin Endocrinol Metab. 2002;87:978-982.

20. Hellman R. Patient safety and inpatient glycemic control: translating concepts into action. Endocr Pract. 2006;12 (Suppl 3):49-55.

21. Cook CB, Castro JC, Schmidt RE, et al. Diabetes care in hospitalized noncritically ill patients: more evidence for clinical inertia and negative therapeutic momentum. J Hosp Med. 2007;2:203-211.

22. Nazer LH, Chow SL, Moghissi ES. Insulin infusion protocols for critically ill patients: a highlight of differences and similarities. Endocr Pract. 2007;13:137-146.

23. Wilson M, Weinreb J, Hoo GW. Intensive insulin therapy in critical care: a review of 12 protocols. Diabetes Care. 2007; 30:1005-1011.

24. Institute for Safe Medication Practices. ISMP's list of highalert medications. Available at: http://www.ismp.org/Tools/ highalertmedications.pdf. Accessed October 2008.

25. American Association of Clinical Endocrinologists. Patient safety and medical system errors in diabetes and endocrinology consensus conference: position statement. Available at: http://www.aace.com/pub/pdf/guidelines/PatientSafetyPositionStatement.pdf. Accessed October 2008.

26. Baldwin D, Villanueva G, McNutt R, Bhatnagar S. Eliminating inpatient sliding-scale insulin: a reeducation project with medical house staff. Diabetes Care. 2005;28:1008-1011.

27. Braithwaite SS, Buie MM, Thompson CL, et al. Hospital hypoglycemia: not only treatment but also prevention. Endocr Pract. 2004;10 (Suppl 2):89-99.

28. Cook CB, Moghissi E, Joshi R, Kongable GL, Abad VJ. Inpatient point-of-care bedside glucose testing: preliminary data on use of connectivity informatics to measure hospital glycemic control. Diabetes Technol Ther. 2007;9:493-500.

29. Workgroup on Hypoglycemia, American Diabetes Association. Defining and reporting hypoglycemia in diabetes: a report from the American Diabetes Association Workgroup on Hypoglycemia. Diabetes Care. 2005;28:1245-1249.

30. Cook CB, Boyle ME, Cisar NS, et al. Use of continuous subcutaneous insulin infusion (insulin pump) therapy in the hospital setting: proposed guidelines and outcome measures. Diabetes Educ. 2005;31:849-857. 